\title{
Susceptibility of Different Faba Bean Cultivars to Infestation With Cowpea Aphid, Aphis Craccivora (Homoptera :Aphidiade) \\ Ezzat F. EL- Khayat ${ }^{1}$, Tahany R. Abd- El Zaher ${ }^{1}$, Eman O. Hassan ${ }^{2}$, Sayed H. A. Hussein ${ }^{3}$ and Hadeer B. EL- Toukhy ${ }^{1}$ \\ ${ }^{1}$ Plant Protection Dept., Fac. Agric., Benha Univ., Egypt. \\ ${ }^{2}$ Plant diseases Dept., Fac. Agric., Benha Univ., Egypt. \\ ${ }^{3}$ Head of Research, plant protection Research Institute \\ Corresponding author: dr.hadeereltoukhy@gmail.com
}

\begin{abstract}
Susceptibility of four broad bean cultivates (Giza 843, Masr 3, Sakha 4 and Sakha 1) to infestation with cowpea aphid, Aphis craccivora, (Homoptera :Aphidiade) was studied. Sakha 4 occurred the first category in lower infestation with aphid while Masr 3 cultivar was the highest one in attracting aphids individuals. Faba bean plants in season $2014 / 2015$ were more infested than that in the second season 2015/2016.

The plants of Masr 3 cultivar have low content of potassium and high content of chlorophyll which may be make that the most attractive to aphids while the plants of Sakha 4 have more amount of potassium and low of chlorophyll making that lowest attractive cultivar to aphids .
\end{abstract}

Key Words: Faba Bean, Aphid, Aphis Craccivora, Giza 843, Masr 3, Sakha 4 and Sakha 1

\section{Introduction}

Faba bean (Vicia faba L.) is one of the most important economic crops grown in Egypt. Seeds of broad bean has highly protein content, which used either as fresh green vegetable or dried for human and animal feeding (Duke, 1981). Faba bean plants is liable to attack by several insect pests, from the early stage of growth and captaincy through the late development to the post-harvest stage.

Aphids is one of the important insect pests in the field. There are many species are common as major pests of economic plants. For instance, the cowpea aphid, Aphis craccivora, (Homoptera :Aphidiade) is a polyphagus pest species of field crops especially of farm leguminosae. It damages the plants by sucking the sap. It also considered a vector of about 30 plants virus diseases. (Blackman and Eastop, 1984: Saenz et al., 2001 and El Hawary and Abd El-Salam, 2005).

The control of insects is difficult because of their small size, high reproduction rates and rapid development of resistance to many insecticides.

This research aimed to study the relationship between phytochemical composition and anatomical characteristics of four faba bean cultivars (Giza 843, Masr 3, Sakha 4 and Sakha 1) with their susceptibility to infestation with Aphids .

\section{Material and Methods}

Susceptibility of certain broad bean cultivars to the infestation with, Aphis craccivora .

\section{Preparetion of field experiment}

This experiment was conducted at the experiment, at the farm of Faculty of Agriculture, Moshtohor, Qalubia Governorate thought two successive growing seasons 2014-2015 and 2015-2016.The chosen area about $126^{\mathrm{m} 2}$ was cultivated with four broad bean cultivated (Giza 843, Masr 3, Sakha 4 and Sakha 1) at Nov. $15^{\text {th }}$. The whole area was divided into 12 replicates (of about $105 \mathrm{~m}^{2}$ ). All replicates were arranged in a randomized complete blocks design. All normal agricultural operation were carried out except for the use of pesticides. Three weeks after planting and for 16 weeks samples of 15 leaves representing all plant levels were weekly collected .10 plants were randomly taken from each replicate (450 leaves from each cultivate). The collected leaves were placed in paper bags and transferred directly to laboratory. All individuals of aphids were counted by using stereomicroscope.

2- Determination of certain phytochemical components and enzymes in different faba bean cultivar leaves and their relation to Aphis craccivora insect pests infestation.

Leaf samples were collected during the vegetative stages. Leaves of each sample were cleaned and washed with distilled water, then quickly dried by placing at gently between filter papers to remove the excess of water. The fresh weight of leaves was determined. The leaves were placed in a drying oven at $60^{\circ} \mathrm{C}$ for one day until constant weight. The dried leaves were crushed by the aid of homogenizer to fine powder and stored in glass bottles to determine carbohydrates and total protein contents according to the methods of Pregl (1945) and Michel et al. (1956). The percentages of reduced and non-reduced sugars were also estimated in the dry powder using the method of Forsee (1938). Also, the phosphorous content was determined according to method of Troug and Meyer (1939) and also (nitrogen, potassium and phenols) and also chlorophyll and Enzymes (estrases, phenoloxidase and peroxidase ).

\section{3- Measurement of anatomical characterstics of four faba bean cultivar leaves. \\ This experiment was conducted to explain the relative susceptibility of the previous four faba bean cultivars with infestation with aphids, jassed, whitefly and leafminers according to the changes in their}


histological characters. Samples of each cultivar leaves were picked up and fixed in FAA solution. Permanent transverse sections for the leaves of the different cultivars were done according to Jackson (1976).

Upper epidermal cuticle thickness, lower epidermal cuticle thickness, upper epidermal thickness, lower epidermal cuticle thickness, No. of palisade tissue layers, palisade tissue thickness, No. of spongy tissue layers, spongy tissue thickness, thickness of collenchyma layers below the upper epidermis at midrib, thickness of upper fibers in vascular bundle, thickness of lower fibers in vascular bundle, thickness of xylem in vascular bundle and thickness of widest xylem vessel were measured.

\section{Statistical analysis}

The collected data were subjected to proper statistical analysis of (F) test according to compare between means L.S.D at .0 .05 level of probability was used according to Duncan (1955).

\section{Results and discussion}

\section{1- Popualation density of Aphis craccivora:}

Numbers of A. craccivora individuals on the four faba bean cultivars (Giza 843, Masr 3, Sakha 4 and Sakha 1) were counted during the two successive season 2014-2015 and 2015-2016. The regular weekly inspection were carried out by estmiting the number of Aphids / 10 plants. The beginning of inspection was on December $6^{\text {th }}$ till the last inspection in March 21 during the two seasons of inspections.

Data obtained and recorded in (Tables, 1) showed that the infestation with $A$. craccivora began with the appearance of plant seedlings. The infestation appeared and counted in small numbers and increased gradually till reached the highest number in the third inspections for the two seasons.

Statistical analysis of the obtained data showed that there were significant differences between all impactions through the period of experiment. The third inspection denote that the plants of faba bean were more infested with aphids on December 20 for the two season of study.

As for the infestation of the different cultivars, data in table (1) showed that Masr 3 cultivar was the most infested one recording seasonal mean number 320 followed by Giza 843 cultivar 176 , Sakha 1159 and finally Sakha 4132 for the first season 2014/2015.

The same trend was recorded in the second season with low numbers for all cultivars recording 187, 109, 100, 79 individuals for Masr 3, Giza 843, Sakha 1 and Sakha 4 respectively.

Statistical analysis showed significant differences between all tested cultivars from the side of infestation with faba bean aphids. Masr 3 variety was the first infested one or the first attractive cultivar followed by Giza 843and Sakha 1 but Sakha 4 cultivar was the lowest extractive one to aphids.

Table 1. No of aphids on Faba bean plant of different cultivars during 2014-2015 and 2015-2016 seasons in Qalubia .

\begin{tabular}{|c|c|c|c|c|c|c|c|c|c|c|c|c|}
\hline \multirow[b]{2}{*}{$\begin{array}{l}\text { Period } \\
\text { (week) }\end{array}$} & \multicolumn{4}{|c|}{ First season } & \multicolumn{4}{|c|}{ Second season } & \multicolumn{4}{|c|}{ Mean of the two seasons } \\
\hline & $\begin{array}{c}\text { Giza } \\
843\end{array}$ & $\begin{array}{c}\text { Masr } \\
3\end{array}$ & $\begin{array}{c}\text { Sakha } \\
1\end{array}$ & $\begin{array}{c}\text { Sakha } \\
4\end{array}$ & $\begin{array}{c}\text { Giza } \\
843\end{array}$ & $\begin{array}{c}\text { Masr } \\
3\end{array}$ & $\begin{array}{c}\text { Sakha } \\
1\end{array}$ & $\begin{array}{c}\text { Sakha } \\
4\end{array}$ & $\begin{array}{c}\text { Giza } \\
843\end{array}$ & $\begin{array}{c}\text { Masr } \\
3\end{array}$ & $\begin{array}{c}\text { Sakha } \\
1\end{array}$ & $\begin{array}{c}\text { Sakha } \\
4\end{array}$ \\
\hline $1^{\text {st }}$ & $79^{1}$ & $68 n$ & $77 \mathrm{k}$ & $60 \mathrm{i}$ & 32 & 38 & 25 & 23 & 55 & 53 & 51 & 42 \\
\hline $2^{\text {nd }}$ & $175 \mathrm{f}$ & $232 \mathrm{i}$ & $127 f$ & $99 \mathrm{~h}$ & 58 & 45 & 53 & 41 & 117 & 139 & 90 & 70 \\
\hline $3^{\text {rd }}$ & 261d & $922 \mathrm{a}$ & $292 c$ & $151 d$ & 84 & 52 & 72 & 63 & 173 & 487 & 182 & 107 \\
\hline $4^{\text {th }}$ & $292 c$ & $328 \mathrm{~g}$ & $247 d$ & $242 b$ & 56 & 50 & 48 & 24 & 174 & 189 & 147 & 133 \\
\hline $5^{\text {th }}$ & $126 \mathrm{ij}$ & 1531 & $118 \mathrm{gh}$ & $141 \mathrm{e}$ & 39 & 80 & 19 & 13 & 82 & 117 & 68 & 77 \\
\hline $6^{\text {th }}$ & $337 \mathrm{a}$ & $399 \mathrm{e}$ & $313 b$ & $272 a$ & 52 & 62 & 42 & 21 & 195 & 231 & 178 & 147 \\
\hline $7^{\text {th }}$ & $263 d$ & $279 \mathrm{~h}$ & $248 d$ & $203 c$ & 61 & 77 & 71 & 30 & 162 & 178 & 160 & 117 \\
\hline $8^{\text {th }}$ & $343 a$ & $427 d$ & $333 a$ & $246 b$ & $90 a$ & 93 & 60 & 28 & 217 & 260 & 197 & 137 \\
\hline $9^{\text {th }}$ & $133 i$ & $479 c$ & 111hi & $120 \mathrm{f}$ & 65 & 35 & 34 & 50 & 99 & 257 & 73 & 85 \\
\hline $10^{\text {th }}$ & $90 \mathrm{k}$ & $380 f$ & $80 \mathrm{k}$ & $56 \mathrm{ij}$ & 49 & 61 & 57 & 40 & 70 & 220 & 69 & 48 \\
\hline $11^{\text {th }}$ & $142 \mathrm{~h}$ & $230 \mathrm{i}$ & $100 \mathrm{j}$ & $58 \mathrm{i}$ & 32 & 57 & 52 & 27 & 87 & 144 & 76 & 43 \\
\hline $12^{\text {th }}$ & $151 \mathrm{~g}$ & $193 k$ & $123 \mathrm{fg}$ & $154 d$ & 22 & 43 & 40 & 30 & 87 & 118 & 82 & 92 \\
\hline $13^{\text {th }}$ & $188 \mathrm{e}$ & $657 b$ & $187 \mathrm{e}$ & $126 f$ & 20 & 74 & 33 & 28 & 104 & 366 & 110 & 77 \\
\hline $14^{\text {th }}$ & $125 \mathrm{j}$ & $215 j$ & $105 \mathrm{ij}$ & $107 \mathrm{~g}$ & 10 & 49 & 26 & 0 & 68 & 132 & 66 & 53 \\
\hline $15^{\text {th }}$ & 721 & $110 \mathrm{~m}$ & 601 & $50 \mathrm{j}$ & 0 & 30 & 10 & 0 & 36 & 70 & 35 & 25 \\
\hline $16^{\text {th }}$ & $37 \mathrm{~m}$ & 540 & $22 \mathrm{~m}$ & $21 \mathrm{k}$ & 0 & 20 & 5 & 0 & 18 & 37 & 13 & 11 \\
\hline Mean & 176 & 320 & 159 & 132 & 42 & 54 & 41 & 26 & 109 & 187 & 100 & 79 \\
\hline $\begin{array}{r}\mathrm{LSD} \text { a } \\
\mathrm{fc}\end{array}$ & 0.05 & & & 7.4 & & & 3.7 & & & & & \\
\hline
\end{tabular}


From the previous results in Table (1) it data can be scemmarized in the following, the infestation with aphid Aphis craccivora was higher in the first season $2014 / 2015$ than that in the second season for all studied cultivars. The infestation began rare and increased till reached the highest infestation at the third inspection (20 of December).
Sakha 4 cultivar was the lowest infested one followed by Sakha 1, Giza 843 but Masr 3 cultivar was the most infested one by attracting the highest number of aphids.

The reason of variation in infestation with aphids of the four cultivars was unknown, because of that it is in need to the following step.

Table 2. Phytochemical screening to the components and certain enzymes of fabae bean cultivars and their effect on aphids infestation.

\begin{tabular}{|c|c|c|c|c|c|c|c|c|c|c|c|c|c|}
\hline \multirow[b]{3}{*}{$\begin{array}{l}\text { Culti } \\
\text { vars }\end{array}$} & \multirow{3}{*}{$\begin{array}{c}\text { Pes } \\
\text { t } \\
\text { Aphi } \\
\text { ds }\end{array}$} & \multicolumn{12}{|c|}{ Correlation coefficient factors } \\
\hline & & \multicolumn{8}{|c|}{ Phytochemical components } & \multirow[b]{2}{*}{$\begin{array}{c}\text { Chloro } \\
\text { phyll }\end{array}$} & \multicolumn{3}{|c|}{ Enzymes } \\
\hline & & $\begin{array}{c}\text { T.p } \\
\cdot\end{array}$ & $\begin{array}{c}\text { Car } \\
.\end{array}$ & R.s. & N.s. & $\mathbf{N}$ & $\mathbf{P}$ & $\mathbf{K}$ & Ph. & & $\begin{array}{l}\text { Estr } \\
\text { uses }\end{array}$ & $\begin{array}{c}\text { Phe } \\
\text { nol } \\
\text { oxid } \\
\text { ase }\end{array}$ & $\begin{array}{c}\text { Peroxi } \\
\text { dase }\end{array}$ \\
\hline $\begin{array}{c}\text { Giza } \\
843\end{array}$ & $4.19^{\mathrm{a}}$ & $\begin{array}{l}4.5 \\
1^{\mathrm{c}}\end{array}$ & $\begin{array}{l}19 . \\
78^{c}\end{array}$ & $\begin{array}{c}8.5 \\
6^{\mathrm{b}}\end{array}$ & $\begin{array}{l}10 . \\
53^{c}\end{array}$ & $728^{c}$ & $\begin{array}{c}177 . \\
3^{\mathrm{a}}\end{array}$ & $\begin{array}{r}122 . \\
13^{c}\end{array}$ & $\begin{array}{l}5.8 \\
8^{c}\end{array}$ & $22.83 b$ & $\begin{array}{c}71.80 \\
b\end{array}$ & $\begin{array}{c}4.27 \\
\mathrm{~d}\end{array}$ & $3.07 \mathrm{c}$ \\
\hline $\begin{array}{c}\text { Masr } \\
3\end{array}$ & $5.42^{\mathrm{a}}$ & $\begin{array}{l}5.1 \\
7^{\mathrm{a}}\end{array}$ & $\begin{array}{l}24 . \\
90^{\mathrm{b}}\end{array}$ & $\begin{array}{c}8.4 \\
1^{\mathrm{b}}\end{array}$ & $\begin{array}{l}15 . \\
83^{\mathrm{b}}\end{array}$ & $\begin{array}{c}810 . \\
30^{\mathrm{a}}\end{array}$ & $\begin{array}{c}144 . \\
00^{\mathrm{b}}\end{array}$ & $\begin{array}{c}104 . \\
6^{\mathrm{d}}\end{array}$ & $\begin{array}{c}5.8 \\
4^{\mathrm{c}}\end{array}$ & $26.92 \mathrm{a}$ & $\begin{array}{c}131.5 \\
0 \mathrm{a}\end{array}$ & $\begin{array}{c}5.46 \\
\mathrm{c}\end{array}$ & $3.90 \mathrm{~b}$ \\
\hline $\begin{array}{c}\text { Sakha } \\
1\end{array}$ & $4.07^{\mathrm{a}}$ & $\begin{array}{l}4.7 \\
3^{b}\end{array}$ & $\begin{array}{l}24 . \\
87^{\mathrm{b}}\end{array}$ & $\begin{array}{c}6.8 \\
7^{c}\end{array}$ & $\begin{array}{l}19 . \\
07^{\mathrm{a}}\end{array}$ & $\begin{array}{l}771 . \\
30^{\mathrm{b}}\end{array}$ & $\begin{array}{c}102 . \\
00^{\mathrm{d}}\end{array}$ & $\begin{array}{c}136 . \\
07^{\mathrm{b}}\end{array}$ & $\begin{array}{c}7.4 \\
5^{\mathrm{b}}\end{array}$ & $23.54 b$ & $\begin{array}{c}74.17 \\
\text { b }\end{array}$ & $\begin{array}{c}6.07 \\
\mathrm{a}\end{array}$ & $4.67 \mathrm{a}$ \\
\hline $\begin{array}{c}\text { Sakha } \\
4\end{array}$ & $2.60_{b}$ & $\begin{array}{l}4.8 \\
9^{b}\end{array}$ & $\begin{array}{l}33 . \\
13^{\mathrm{a}}\end{array}$ & $\begin{array}{l}13 . \\
81^{\mathrm{a}}\end{array}$ & $\begin{array}{l}20 . \\
83^{a}\end{array}$ & $\begin{array}{l}787 . \\
70^{\mathrm{b}}\end{array}$ & $\begin{array}{c}127 . \\
00^{c}\end{array}$ & $\begin{array}{c}153 . \\
3^{\mathrm{a}}\end{array}$ & $\begin{array}{c}8.8 \\
9^{a}\end{array}$ & $20.05 \mathrm{c}$ & $\begin{array}{c}75.50 \\
\text { b }\end{array}$ & $\begin{array}{c}5.86 \\
b\end{array}$ & $4.03 \mathrm{~b}$ \\
\hline $\mathrm{F}$ & 9.88 & $\begin{array}{l}20 . \\
37\end{array}$ & $\begin{array}{l}16 . \\
15\end{array}$ & $\begin{array}{l}49 . \\
71\end{array}$ & $\begin{array}{c}8.3 \\
9\end{array}$ & 9.60 & $\begin{array}{c}18.5 \\
1\end{array}$ & $\begin{array}{c}14.2 \\
9\end{array}$ & $\begin{array}{l}23 . \\
36\end{array}$ & 18.7 & 23.92 & $\begin{array}{c}34.0 \\
9\end{array}$ & 36.57 \\
\hline L.S.D & 1.96 & $\begin{array}{c}0.2 \\
0\end{array}$ & $\begin{array}{c}4.4 \\
8 \\
\end{array}$ & $\begin{array}{c}1.4 \\
0\end{array}$ & $\begin{array}{c}3.1 \\
2\end{array}$ & $\begin{array}{c}19.6 \\
0\end{array}$ & 7.18 & $\begin{array}{c}11.2 \\
3\end{array}$ & $\begin{array}{c}0.9 \\
8\end{array}$ & 1.5 & 12.83 & 0.23 & 0.22 \\
\hline $\mathrm{R}$ & & 0.35 & -0.66 & -0.75 & -0.51 & 0.19 & 0.26 & -0.61 & -0.84 & 0.98 & 0.15 & -0.47 & -0.19 \\
\hline
\end{tabular}

1. Correlation between phytochemical components in the four faba bean varieties and the infestation means of Aphid caccivora

To explain and show the reason of variation in the different faba bean cultivars infestation with aphids the phytochemical components and leaf layer thickness were estimated.

1- The relationship between phytochemical components and the infestation with aphids .

Eight phytochemical components (total proteins, total carbohydrates, reducing sugars, non-reducing sugars, nitrogen, phosphorus, potassium and phenols) and also chlorophyll and Enzymes (estrases , phenoloxidase and peroxidase ) were determined (table 2). Data showed that there were significant differences in $\mathrm{K}$ (potassium amount, chlorophyll and estrases enzymes between the most infested cultivar ( Masr 3) and the lowest infested one (Sakha 4).
Potassium amount was more in Sakha 4 cultivar (153.3) and the plants of the same cultivar have low chlorophyll amount $(20,5)$ than the other cultivars . Also, Masr3 cultivar has more amount of estrases enzymes (131.50). Because of the significant differences in the contents of the four cultivars with these three components the infestation with aphids varied from one to another cultivars. Potasium helps in the formation of solid tissues in leaves which make it difficult to the mouth parts of aphid to absorb plant guise . Also the increase of chlorophyll in Masr3 cultivar encourage and attract aphids to its plants. Also the increase of estrastes enzymes which a hydrolase enzymes that splits esters into acid and an alcohol and make plants more edible and help as carminative and help insects in remedy them from dizzeness (Napat $\mathrm{T}$. et al .2013) 
Table 3. Relation between leaf layers thickness of four fabae bean and aphids infestation throughout second season 2015-2016

\begin{tabular}{|c|c|c|c|c|c|c|c|c|c|c|c|c|c|c|}
\hline \multirow[b]{2}{*}{ 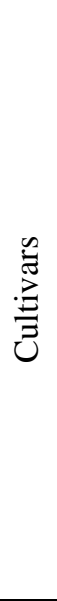 } & \multirow[b]{2}{*}{$\stackrel{\mathbb{E}}{\Sigma}^{\mathbb{E}}$} & \multicolumn{13}{|c|}{ Leaf layers } \\
\hline & & $\begin{array}{c}\text { Upp } \\
\text { er } \\
\text { epid } \\
\text { erma } \\
1 \\
\text { cutic } \\
\text { le } \\
\text { thick } \\
\text { ness. }\end{array}$ & $\begin{array}{c}\text { Low } \\
\text { er } \\
\text { epid } \\
\text { erma } \\
1 \\
\text { cutic } \\
\text { le } \\
\text { thick } \\
\text { ness. }\end{array}$ & $\begin{array}{l}\text { Upp } \\
\text { er } \\
\text { epid } \\
\text { erma } \\
1 \\
\text { thick } \\
\text { ness. }\end{array}$ & $\begin{array}{c}\text { Low } \\
\text { er } \\
\text { epid } \\
\text { erma } \\
1 \\
\text { cutic } \\
\text { le } \\
\text { thick } \\
\text { ness. }\end{array}$ & $\begin{array}{c}\text { No. } \\
\text { of } \\
\text { pali } \\
\text { sad } \\
\text { e } \\
\text { tiss } \\
\text { ue } \\
\text { laye } \\
\text { rs. }\end{array}$ & $\begin{array}{l}\text { Palis } \\
\text { ade } \\
\text { tissu } \\
\text { e } \\
\text { thick } \\
\text { ness. }\end{array}$ & $\begin{array}{c}\text { No. } \\
\text { of } \\
\text { spo } \\
\text { ngy } \\
\text { tiss } \\
\text { ue } \\
\text { lay } \\
\text { ers. }\end{array}$ & $\begin{array}{l}\text { Spon } \\
\text { gy } \\
\text { tissu } \\
\text { e } \\
\text { thick } \\
\text { ness. }\end{array}$ & $\begin{array}{l}\text { Thick } \\
\text { ness } \\
\text { of } \\
\text { collen } \\
\text { chym } \\
\text { a } \\
\text { layers } \\
\text { below } \\
\text { the } \\
\text { upper } \\
\text { epider } \\
\text { mis at } \\
\text { midri } \\
\text { b. }\end{array}$ & $\begin{array}{c}\text { Thic } \\
\text { knes } \\
\mathrm{s} \text { of } \\
\text { uppe } \\
\mathrm{r} \\
\text { fiber } \\
\mathrm{s} \text { in } \\
\text { vasc } \\
\text { ular } \\
\text { bund } \\
\text { le. }\end{array}$ & $\begin{array}{c}\text { Thic } \\
\text { knes } \\
\text { s of } \\
\text { lowe } \\
\text { r } \\
\text { fiber } \\
\text { s in } \\
\text { vasc } \\
\text { ular } \\
\text { bund } \\
\text { le. }\end{array}$ & $\begin{array}{c}\text { Thic } \\
\text { knes } \\
\text { s of } \\
\text { xyle } \\
\text { m in } \\
\text { vasc } \\
\text { ular } \\
\text { bund } \\
\text { le. }\end{array}$ & $\begin{array}{c}\text { Thic } \\
\text { knes } \\
\text { s of } \\
\text { wide } \\
\text { st } \\
\text { xyle } \\
\mathrm{m} \\
\text { vess } \\
\text { el in } \\
\text { vasc } \\
\text { ular } \\
\text { bund } \\
\text { le }\end{array}$ \\
\hline $\begin{array}{c}\mathrm{Gi} \\
\mathrm{za} \\
84 \\
3 \\
\end{array}$ & $\begin{array}{c}4 . \\
1 \\
9\end{array}$ & $\begin{array}{c}10.8 \\
0^{\mathrm{b}}\end{array}$ & $8.10^{\mathrm{a}}$ & $\begin{array}{c}25.2 \\
0^{\mathrm{b}}\end{array}$ & $\begin{array}{c}13.5 \\
0^{\mathrm{b}}\end{array}$ & $\begin{array}{c}1.0 \\
0^{\mathrm{a}}\end{array}$ & $\begin{array}{c}45.0 \\
0^{c}\end{array}$ & $\begin{array}{c}5.0 \\
0^{\mathrm{a}}\end{array}$ & $\begin{array}{c}90.0 \\
0^{\mathrm{b}}\end{array}$ & $9.00^{\mathrm{b}}$ & $\begin{array}{c}45.0 \\
0^{\mathrm{d}}\end{array}$ & $\begin{array}{c}85.5 \\
0^{c}\end{array}$ & $\begin{array}{c}112 . \\
50^{\mathrm{d}}\end{array}$ & $\begin{array}{c}13.5 \\
0^{\mathrm{d}}\end{array}$ \\
\hline $\begin{array}{c}\mathrm{M} \\
\text { asr } \\
3 \\
\end{array}$ & $\begin{array}{l}5 . \\
4 \\
2 \\
\end{array}$ & $8.10^{c}$ & $6.30^{\mathrm{b}}$ & $\begin{array}{c}18.0 \\
0^{\mathrm{d}}\end{array}$ & $\begin{array}{c}10.8 \\
0^{c}\end{array}$ & $\begin{array}{c}1.0 \\
0^{\mathrm{a}}\end{array}$ & $\begin{array}{c}38.7 \\
0^{\mathrm{d}}\end{array}$ & $\begin{array}{c}5.0 \\
0^{\mathrm{a}}\end{array}$ & $\begin{array}{c}90.0 \\
0^{\mathrm{b}}\end{array}$ & $0.00^{c}$ & $\begin{array}{c}80.1 \\
0^{c}\end{array}$ & $\begin{array}{c}81.0 \\
0^{\mathrm{d}}\end{array}$ & $\begin{array}{c}171 . \\
00^{c}\end{array}$ & $\begin{array}{c}18.0 \\
0^{\mathrm{b}}\end{array}$ \\
\hline $\begin{array}{l}\mathrm{Sa} \\
\mathrm{kh} \\
\mathrm{a} 1 \\
\end{array}$ & $\begin{array}{c}4 . \\
0 \\
7 \\
\end{array}$ & $\begin{array}{c}10.8 \\
0^{\mathrm{b}}\end{array}$ & $8.10^{\mathrm{a}}$ & $\begin{array}{c}22.5 \\
0^{\mathrm{c}}\end{array}$ & $\begin{array}{c}13.5 \\
0^{\mathrm{b}}\end{array}$ & $\begin{array}{c}1.0 \\
0^{\mathrm{a}}\end{array}$ & $\begin{array}{c}49.5 \\
0^{\mathrm{b}}\end{array}$ & $\begin{array}{c}5.0 \\
0^{\mathrm{a}}\end{array}$ & $\begin{array}{c}85.5 \\
0^{c}\end{array}$ & $13.50^{\mathrm{a}}$ & $\begin{array}{c}86.4 \\
0^{\mathrm{b}}\end{array}$ & $\begin{array}{c}117 . \\
00^{\mathrm{b}}\end{array}$ & $\begin{array}{c}175 . \\
50^{\mathrm{b}}\end{array}$ & $\begin{array}{c}16.2 \\
0^{c}\end{array}$ \\
\hline $\begin{array}{l}\mathrm{Sa} \\
\mathrm{kh} \\
\mathrm{a} 4\end{array}$ & $\begin{array}{c}2 . \\
6 \\
0\end{array}$ & $\begin{array}{c}16.2 \\
0^{\mathrm{a}}\end{array}$ & $9.00^{\mathrm{a}}$ & $\begin{array}{c}28.8 \\
0^{\mathrm{a}}\end{array}$ & $\begin{array}{c}18.0 \\
0^{\mathrm{a}}\end{array}$ & $\begin{array}{c}1.0 \\
0^{\mathrm{a}}\end{array}$ & $\begin{array}{c}76.5 \\
0^{\mathrm{a}}\end{array}$ & $\begin{array}{c}5.0 \\
0^{\mathrm{a}}\end{array}$ & $\begin{array}{c}130 . \\
50^{\mathrm{a}}\end{array}$ & $9.00^{\mathrm{b}}$ & $\begin{array}{c}112 . \\
50^{\mathrm{a}}\end{array}$ & $\begin{array}{c}189 . \\
00^{\mathrm{a}}\end{array}$ & $\begin{array}{c}249 . \\
30^{\mathrm{a}}\end{array}$ & $\begin{array}{c}19.8 \\
0^{\mathrm{a}}\end{array}$ \\
\hline $\mathrm{F}$ & $\begin{array}{l}9 . \\
8 \\
8\end{array}$ & $\begin{array}{c}41.9 \\
7\end{array}$ & $\begin{array}{c}14.7 \\
4\end{array}$ & $\begin{array}{c}44.5 \\
6\end{array}$ & $\begin{array}{c}79.2 \\
0\end{array}$ & $\begin{array}{c}0.0 \\
0\end{array}$ & $\begin{array}{c}578 . \\
81\end{array}$ & $\begin{array}{c}0.0 \\
0\end{array}$ & $\begin{array}{c}663 . \\
28\end{array}$ & $\begin{array}{c}127.8 \\
2\end{array}$ & $\begin{array}{c}1097 \\
.38\end{array}$ & $\begin{array}{c}4779 \\
.00\end{array}$ & $\begin{array}{c}3016 \\
.25\end{array}$ & $\begin{array}{c}50.9 \\
8\end{array}$ \\
\hline $\begin{array}{l}\text { L. } \\
\text { S. } \\
\text { D }\end{array}$ & $\begin{array}{l}1 . \\
9 \\
5\end{array}$ & 1.71 & 0.94 & 2.22 & 1.09 & $\begin{array}{c}0.0 \\
0\end{array}$ & 1.01 & $\begin{array}{c}0.0 \\
0\end{array}$ & 2.67 & 1.63 & 2.30 & 2.35 & 3.32 & 1.23 \\
\hline $\mathrm{R}$ & & $\begin{array}{c}- \\
0.30\end{array}$ & $\begin{array}{c}- \\
0.61\end{array}$ & $\begin{array}{c}- \\
0.36\end{array}$ & $\begin{array}{c}- \\
0.35\end{array}$ & $\begin{array}{c}0.0 \\
0 \\
\end{array}$ & $\overline{-}-26$ & $\begin{array}{c}0.0 \\
0\end{array}$ & 0.09 & -0.96 & -0.14 & -0.30 & -0.70 & 0.23 \\
\hline
\end{tabular}




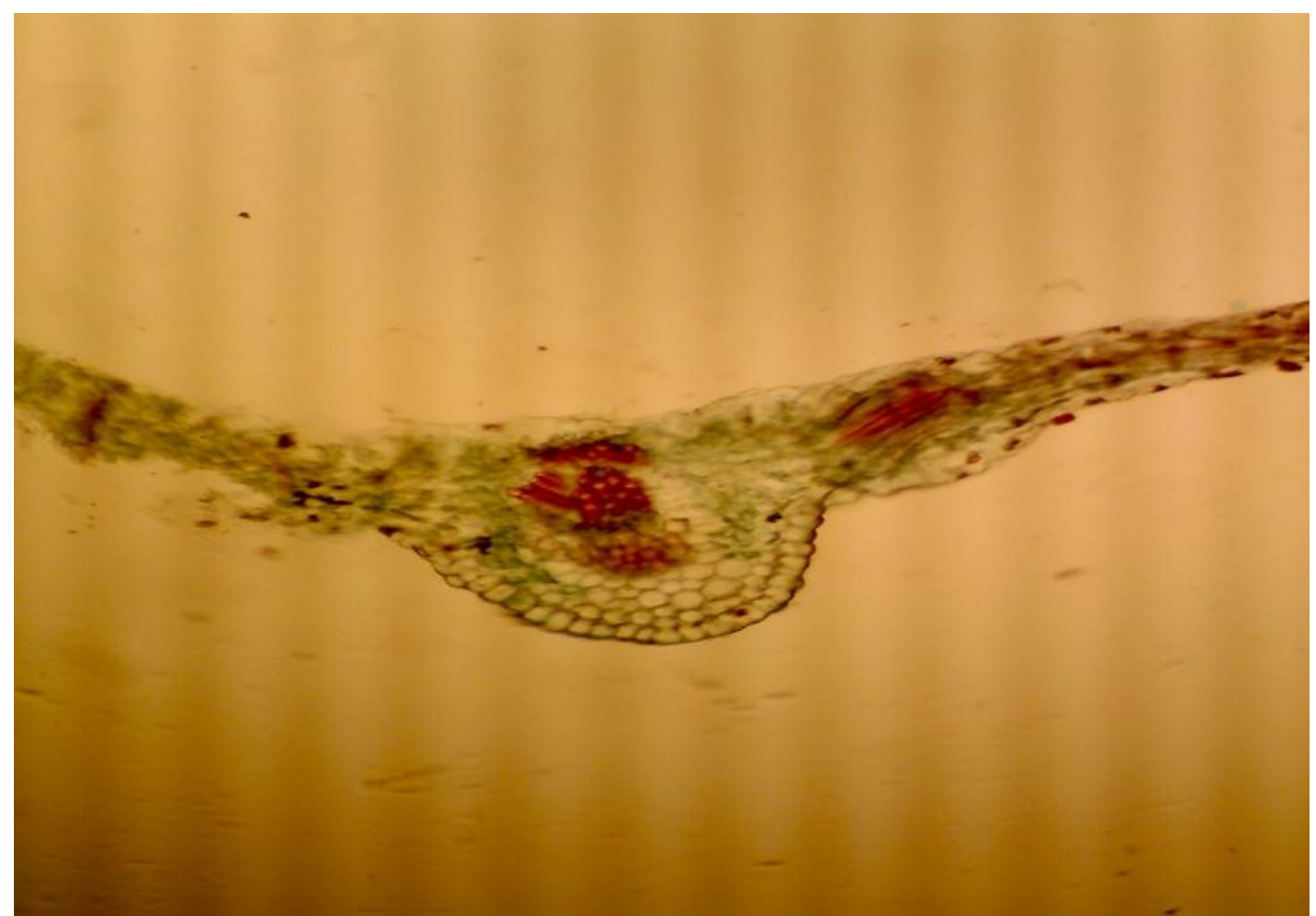

Giza 843

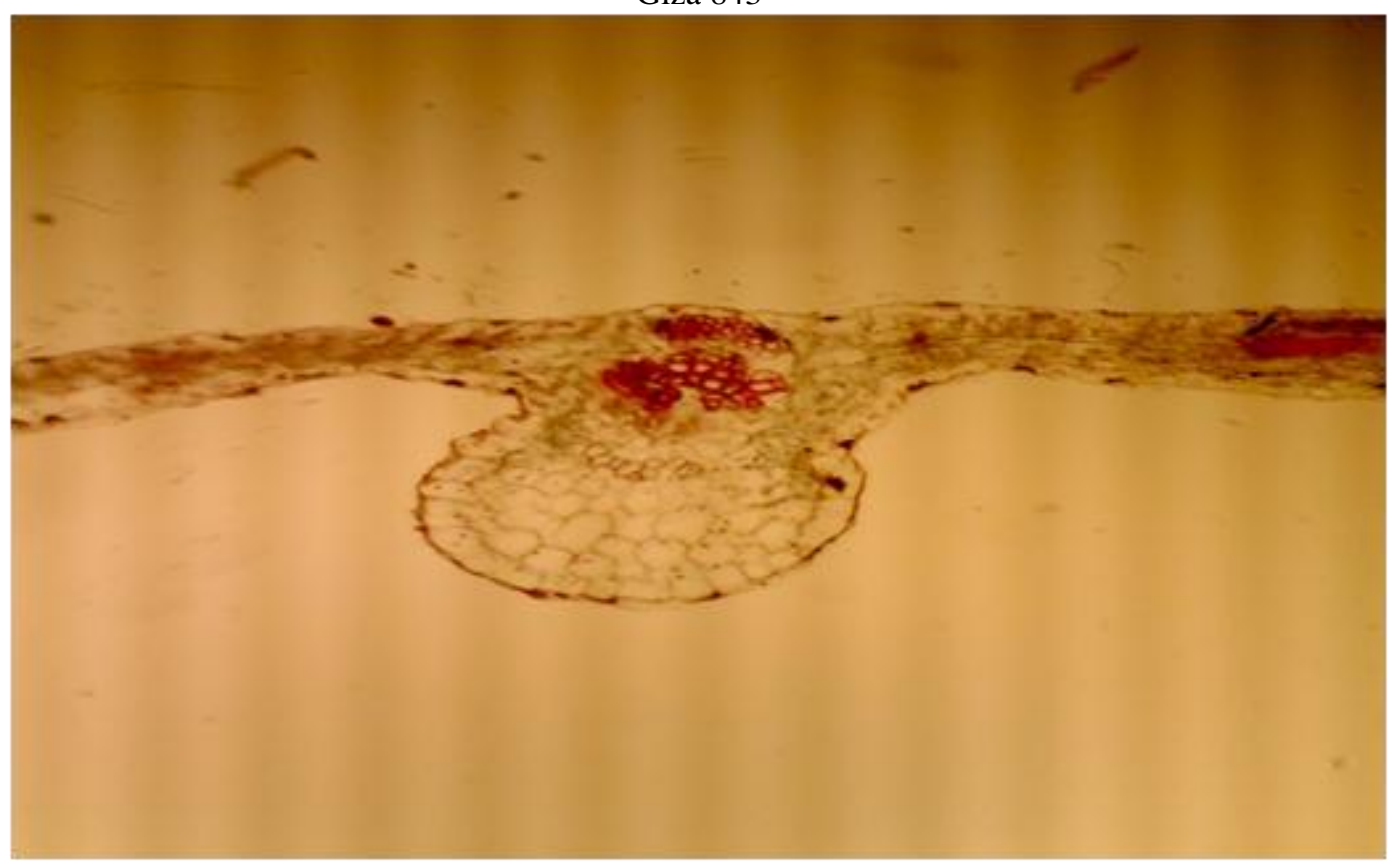

Masr 3 


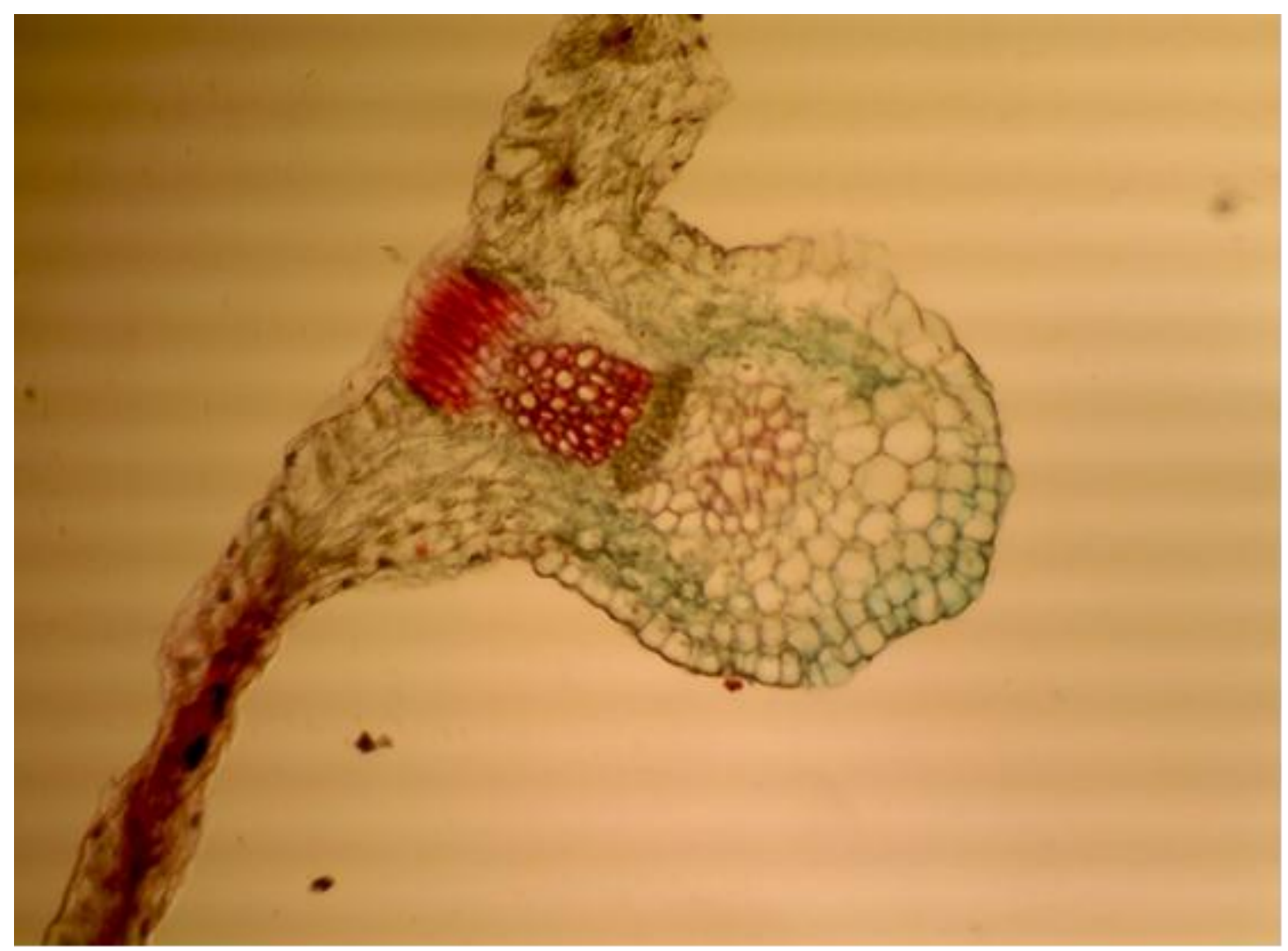

Sakha 1

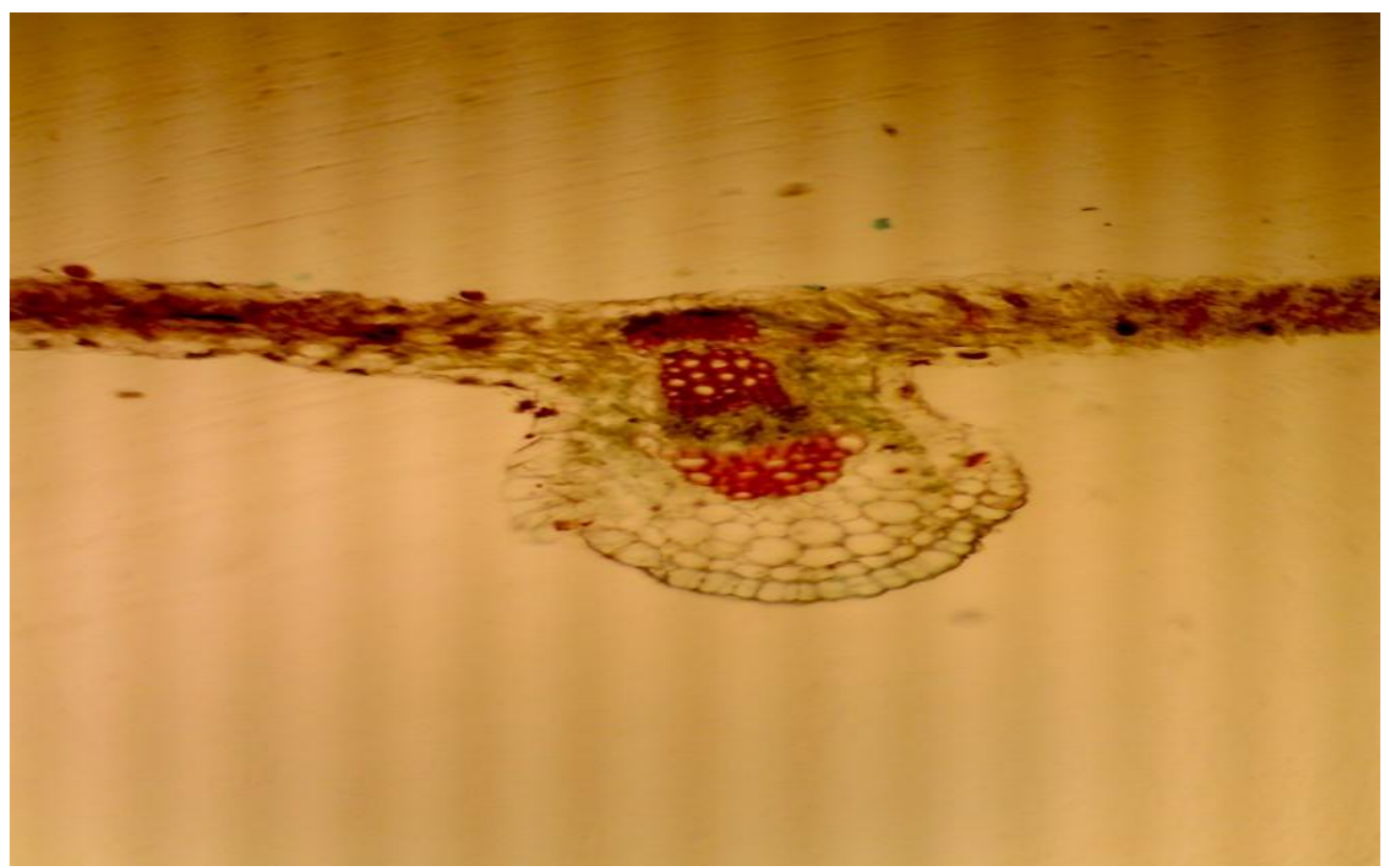

Sakha 4

Fig. (1): Anatomical character of tested faba bean varieties 
2. The relationship between the infestation of faba bean cultivars with aphids and anatomical characteristics of its leaves.

One of the most important factors which explained the degree susceptibility of the previously mentioned faba bean cultivars to infestation of Aphids insects is the effect of anatomical characters. This part of study was done to discover the correlation between the layers of tested leaurs and the population density of insects for the four studied faba bean cultivars at season 2015-2016.

The relation between the thickness of each layer and the population density of certain insect pests can be explained as follows:

1. Aphids (mainly Acyrthosiphon pisum, Myzus persicae and Aphis craccivora).

The seasonal mean counts of aphids infesting leaves of the 4 faba bean cultivars were tabulated in Table with the mean thickness of leaf layers of these cultivars and their correlation.

A significant difference between the mean count of aphids harboured on leaves of the four tested cultivars was found, as the heaviest infestation was recorded on leaves of Masr 3 variety (5.42 individuals/leaflets) which had the thinnest layers of the upper and lower epidermal cuticle thickness, upper and lower epidermal thickness, the palisade tissue thickness, thickness of collenchyma layers below the upper epidermis at midrib and Thickness of lower fibers in vascular bundle $(8.10,6.30,18.00,10.80$, $38.70,0.00$ and 81.00 , respectively), and also average of spongy tissue thickness, thickness of upper fibers in vascular bundle, thickness of xylem in vascular bundle and thickness of widest xylem vessel in vascular bundle $(90.00,80.10,171.00$ and 18.00, respectively), as the lowest infestation was recorded on leaves of Sakha 4 cultivar (2.60 individuals/leaflet) which had the thinnest larger of the upper and lower epidermal cuticle thickness, upper and lower epidermal thickness, the palisade tissue thickness, Spongy tissue thickness, thickness of upper fibers in vascular bundle, thickness of lower fibers in vascular bundle, thickness of xylem in vascular bundle and thickness of widest xylem vessel in vascular bundle $(16.20,9.00,28.80,18.00,76.50,130.50,112.50$, $189.00,249.30$ and 19.80 , respectively) and also the thickest spongy layer (9.00).

On the other hand, the other two species were average in the aphids infection and were therefore average in the thickness of the layers, Giza 843 and Sakha 1 were the injury rate (4.19 and 4.07) and therefore were the upper and lower epidermal cuticle thickness, upper and lower epidermal thickness, the palisade tissue thickness, spongy tissue thickness, thickness of collenchyma layers below the upper epidermis at midrib, thickness of upper fibers in vascular bundle, thickness of lower fibers in vascular bundle, thickness of xylem in vascular bundle and Thickness of widest xylem vessel in vascular bundle
$(10.80,8.10,25.20,13.50,45.00,90.00,9.00,45.00$, $85.50,112.50$ and 13.50, respectively ) so in Giza 843 cultivar, Bat in Sakha $1(10.80,8.10,22.50,13.50$, $49.00,85.50,13.50,86.40,117.00,175.50$ and 16.20).

From the same table, the correlation coefficient (r) values were insignificantly positive for the relationship between aphid populations and Spongy tissue thickness and the thickness of widest xylem vessel in vascular bundle of the leaf (0.09 and 0.23], respectively). Also these values were insignificantly negative for the the upper and lower epidermal cuticle thickness, upper and lower epidermal thickness, the palisade tissue thickness, thickness of collenchyma layers below the upper epidermis at midrib, thickness of upper fibers in vascular bundle, thickness of lower fibers in vascular bundle and Thickness of xylem in vascular bundle $(-0.30,-0.61,-0.36,-0.35,-0.26$, $0.96,-0.14,-0.30$ and -0.70 , respectively) (Table, 3 ).

The variations in the susceptibility degrees among the tested faba bean varieties towards the infestation by insects may be due to various factors. These factors include the cultivar used, date of sowing, locality and the environmental conditions prevailing in the area under study particularly, humidity and temperature, as well as presence of antibiosis and/or non-preference (antixenoses) phenomena Mohamed (2011), Mohamed (2003) and Van Emden (1987).

\section{References}

Blackman, R.L.; and Eastop V.F. 1984. Aphids on the world's crops; An identification and information guide. John Wiley \& Sons, Chichester, New York Brisbane, Toronto, Singapore, $463 \mathrm{pp}$.

Duke, J.A.1981. Hand book of legumes of world economic importance. Plenum press, New York.PP:199-265 E

Duncan, D.B.1955. Multiple range and multiple F. tests . Biometrics, 11: 1-24.

El-Hawary, F. M. A.; and Abd EI-Salam, A. M. E. 2005. Insecticidal effects of Fenugreek oil and benzaldehyde (aromatic compounds) on cowpea aphid, Aphis craccivora Koch. J. Egypt. Ger. Soc. Zool, 46E, 1: 43 - 56.

Forsee,W.T. 1938. Determination of sugar in plant material a photo colormetric method .Inds.Eng.Chem.Ann.10th ed.:411-418.

Jackson,G. 1976. Crystal violet and erythrosine in plant anatomy.Stain Tech.1:33-34.

Michel,K.A. ; Gilles .J.K.; Hamilton. P.A.; and Smith .F. 1956. Colorimetric method for determination of sugars and related substances .Analytical Chemistry, 28(3): 302-307.

Mohamed, M. A. A. 2003. Studied on certain insect pests infesting faba bean (Vicia faba L.) with relation to their natural enemies in sohag Upper Egypt. D.Ph. Thesis, plant protection Dept., Assiut Univ., Egypt. 
Mohamed, M. A. 2011. Effect of planting dates on infestations with certain pests and yield parameters of Squash plants. Egypt. J. Agric. Res., 89 (4): 1353-1361.

Napat,T.; Kitphat W.; Sarina T.; Nuntavan B.; and Veena N. 2013. Neurite out growth and Neusoprotective effects of querctin from Caesalpinia mimosoides lamk on cultered pig derived neurons. Evidence - Based complementary and alternative Medicine Vol. 20137 pages .

Pregl, F. 1945. Quantitative organic microanalysis 4thed. J. andA.Chundril 4th .London.94-101.

Sanz, N. T.; Chen, T.; and Lai, P. 2001. A newly discovered mosaic disease of bush basil Ocimum basilicum in Taiwan. Plant Pathology Bulletin, 10 (4): $155-164$.

Tawfeek, M.E. 2001. Studies on certain aphid species infesting some fruit trees. M. Sc.Thesis Fac. Agric. Alex. Univ.: 152 pp.

Troug ,E.; and Meyer .A.H. 1939. Improvement in deiness colorimetric for phosphorus and arsenic .Ind. Eng. Chen.Ann.Ed.,1:136-139.

Van Emden, H. F. 1987. Cultural methods: the plant, PP. 27-67. In: integrated pest management. (eds.) Burn, A. J., T. H. Coaker and P. C. Jepson, Academic Press, London, New York. 